\title{
Dose-volumetric parameters and prediction of severe acute esophagitis in patients with locally-advanced non small-cell lung cancer treated with neoadjuvant concurrent hyperfractionated-accelerated chemoradiotherapy
}

\author{
Farkhad Manapov ${ }^{1 *+}$, Susanna Sepe ${ }^{1 \dagger}$, Maximilian Niyazi ${ }^{1}$, Claus Belka ${ }^{1}$, Godehard Friedel $^{2}$ and Wilfried Budach ${ }^{3}$
}

\begin{abstract}
Background: To identify dose-volume parameters predictive for severity of acute esophagitis (CTC > grade 2) in locally-advanced non small-cell lung cancer (LA-NSCLC) patients treated with neoadjuvant concurrent hyperfractionated-accelerated chemoradiotherapy (HA-CRT) a retrospective analysis was performed. 88 patients were treated with HA-CRT followed by radical surgery. Predictive power of absolute oesophageal length, absolute and relative oesophageal volume included in the 95\%-isodose, patient- and tumor-related factors for severity of acute esophagitis was assessed.

Findings: A total of 82 patients (93\%) developed radiation-induced acute esophagitis. Grade 1 was documented in 1 (1\%), grade 2 in 55 (67\%), grade 3 in 23 (28\%) and grade 4 in $3(4 \%)$ patients, respectively. Absolute oesophageal volume included in the 95\%-isodose (42.8 Gy) achieved $13.5 \mathrm{~cm}^{3}$ (range: $3-29 \mathrm{~cm}^{3}$ ). Of the tested variables in univariate analysis, absolute oesophageal volume included in the $95 \%$-Isodose was found to be the only significant variable $(p=0.03)$ predicting severe acute esophagitis $(C T C>$ grade 2$)$. For this volume a gradation scale of the likelihood of severity was built.

Conclusion: Increase of absolute oesophageal volume included in the 95\%-isodose correlates with severity of acute esophagitis in LA-NSCLC patients treated with neo-adjuvant concurrent HA-CRT.
\end{abstract}

Keywords: Esophagitis, Hyperfractionated accelerated chemoradiotherapy, Lung cancer

\section{Background}

Concurrent chemoradiotherapy (CRT) is the most relevant treatment option for inoperable LA-NSCLC. Metaanalysis documented that definitive concurrent CRT is associated with a significant prolongation of overall survival compared to thoracic irradiation (TRT) alone [1]. However, local control is still poor. Thus, new treatment protocols aiming to increase local control are urgently needed. Increasing the dose density via hyper-fractionated-accelerated radiation protocols represents one of the known options in

\footnotetext{
*Correspondence: farkhad.manapov@med.uni-muenchen.de

${ }^{\dagger}$ Equal contributors

'Klinik und Poliklinik für Strahlentherapie und Radioonkologie, Klinikum der Universität München, München, Germany

Full list of author information is available at the end of the article
}

order to increase local control rates. Saunders et al. were the first who documented a superiority of HA-radiotherapy over conventional treatment regarding local relapse and survival in NSCLC [2]. Similar results were reported for limited-disease small-cell lung cancer treated with CRT [3]. RTOG 9410 phase III randomised trial for LA-NSCLC which compared three different definitive CRT regimens has reported that there were significantly fewer patients with disease progression within the radiation field when treated with concurrent HA-CRT [4].

An even more intense treatment in implemented when a concurrent application of HA-TRT and chemotherapy is followed by radical resection. At least two single-centre trials have applied such a protocol and demonstrated up 
to $60 \%$ of pathological down-staging and significantly improved long-term survival [5-7]. A multicentre randomised phase III trial investigating this tri-modality concept is still ongoing (EsPaTü) [8].

As trade-off, any dose escalation and treatment intensification of TRT generally results in higher toxicity. Besides pneumonitis, acute esophagitis is a highly important adverse effect and represents a dose-limiting toxicity [9]. Therefore, the identification of predictive factors for severe radiation-induced acute esophagitis is of high clinical relevance. Most data concerning this clinical problem reflect the situation in patients treated with conventional TRT and it has to be questioned in how far these data can be applied to HA-CRT. To define relevant parameters in patients with hyper-fractionated tri-modality concepts the impact of several dose-volume parameters, patient- and tumor-related factors on the severity of acute oesophageal reaction in patients with LA-NSCLC were analysed.

\section{Findings}

All 88 patients (median age 55 years for women and 58 years for men) have participated in a prospective phase II trial [5,7], were diagnosed with initially inoperable histologically proven LA-NSCLC and treated with neoadjuvant HA-CRT followed by radical surgery whenever possible. All patients had a performance score (PS) WHO 1-2. LA-NSCLC was defined as a disease corresponding to Stage III A(2)-B after completion of initial staging. All patients provided written informed consent.

Patients received four cycles of induction chemotherapy (weekly carboplatin dosed at AUC 2 and paclitaxel $100 \mathrm{mg} / \mathrm{m}^{2}$ ) followed by 3 weeks of HA-CRT with 1.5 Gy twice a day up to 45 Gy combined with weekly carboplatin AUC 2 and paclitaxel $50 \mathrm{mg} / \mathrm{m}^{2}$. Irradiation was delivered with a linear accelerator using a multiple field technique. 3D-CT treatment planning was performed. Clinical target volume included primary tumor with $1.0-\mathrm{cm}$ safety margin and bilateral mediastinal nodes from the jugulum to the level of the heart valves. Lower mediastinum was included if nodal involvement was suspected. For each patient oesophagus was delineated from cricoid to gastro-oesophageal junction. Total absolute oesophageal length and volume as well as length and volume included in the $95 \%$-isodose $(42.8 \mathrm{~Gy})$ were measured. Dose-volume histograms of oesophagus were provided for each individual patient. Symptom assessment of acute esophagitis was performed weekly during the whole course of HA-CRT. Severity of acute esophagitis was documented according to the "CTC v. 2". Putative correlations between absolute total oesophageal length, length included in the 95\%-isodose, absolute total oesophageal volume, volume included in the $95 \%$-Isodose and relative oesophageal volume within the $95 \%$-Isodose as well as age at diagnosis, gender, initial T-stage, hematotoxicity and severity of the acute oesophageal reaction were analysed using the binary logistic regression module of the "XLSTAT" software package. P-values and asymptotic confidence limits were calculated based on the respective co-variance matrices.

A total of 82 treated patients (93\%) developed acute esophagitis. Grade 1 acute esophagitis was detected in 1 (1\%), grade 2 in 55 (67\%), grade 3 in $23(28 \%)$ and grade 4 in $3(4 \%)$ patients, respectively. All 82 patients received symptomatic care. No esophagogastroduodenoscopy was necessary during the whole course of treatment. Dose-volumetric parameters of oesophagus were correlated to the corresponding CTC-degree of the radiation-induced acute esophagitis for each individual patient. A similar analysis was done for patient- and tumor-related factors.

On average, absolute total oesophageal length was $23.5 \mathrm{~cm}$ (range: $18-28 \mathrm{~cm}$ ) and median length included in the 95\%-isodose (42.8 Gy) reached $13 \mathrm{~cm}$ (range: $6-21 \mathrm{~cm}$ ). Median total oesophageal volume was $24.5 \mathrm{~cm}^{3}$ (range: $9-43 \mathrm{~cm}^{3}$ ). Volume included in the 95\%-isodose (42.8 Gy) achieved on average $13.5 \mathrm{~cm}^{3}$ (range: $3-29 \mathrm{~cm}^{3}$ ) and accounted for $51 \%$ (range: 18 $89 \%)$ of total organ volume. Of the tested variables in the univariate analysis, absolute oesophageal volume included in the $95 \%$-isodose was found to be the only significant variable $(\mathrm{p}=0.03)$ predicting severe acute esophagitis (CTC > grade 2$)$. No events of severe esophagitis were observed at oesophageal volumes in the 95\%-isodose below $10 \mathrm{ml}$. The original data and the resulting logistic regression are shown in Figure 1.

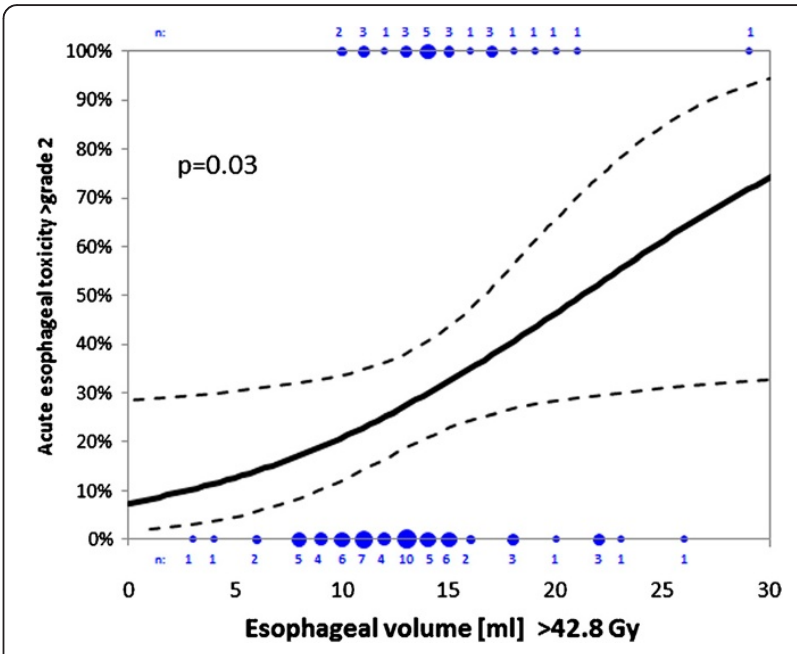

Figure 1 Likelihood of acute severe acute esophageal reaction (>CTC grade 2 ) depends on the absolute esophageal volume within $\mathbf{9 5 \%}$-Isodose (>42.8 Gy). The original binary data and the resulting binary logistic regression line including the $95 \%$ confidence limits (dashed lines) are displayed. Blue symbols and numbers indicate events and number of events at the respective volume. 
No significant correlation for patient- and tumor-related factors and severity of radiation-induced acute esophagitis was found.

\section{Discussion}

Neoadjuvant concurrent HA-CRT followed by radical surgery in LA-NSCLC was shown to be a promising approach in single-centre studies and a multi-institutional randomized phase III trial is actually ongoing [5-8].

In previous trials dose escalation and intensification of TRT resulted in higher acute oesophageal toxicity which was established as dose-limiting in patients treated with different types of CRT. RTOG 9410 trial investigating three different regimens reported a $45 \%$ rate of grade 3 acute esophagitis in the accelerated CRT arm [4]. Earlier, Ball et al. have shown a significant increase of the rate of severe acute esophagitis from $21 \%$ in the conventional on $42 \%$ in accelerated CRT arm [10]. RTOG database analysis on 528 LA-NSCLC patients revealed a strong association of severe acute esophagitis with hyperfractionated CRT [11].

That is why a question arises again whether the severity of acute esophagitis can be predicted on the basis of individual dose-volumetric parameters. Patel et al. reported in accelerated CRT that the critical parameter for development of acute esophagitis was the oesophageal volume treated with $>50$ Gy [12]. A japanese study of conventional CRT from Hirota and colleagues recommended that the absolute oesophageal length and oesophageal volume within 45 Gy-Isodose should amount less than $9.5 \mathrm{~cm}$ and $40 \%$, respectively [13]. Importantly, both studies included only a small number of patients. De Ruyck et al. have found mean oesophageal dose, overall treatment time and radiation technique as important parameters predicting severe acute esophagitis [14].

Present study analysed highest number of patients treated with neoadjuvant concurrent HA-CRT. Our analysis was built on the investigation of individual TRT plans. Importantly, there were no differences in regard to the PS, TNM stage and applied multimodality protocol within the treated population. A comparison of calculated dose-volumetric parameters with carefully assessed individual symptoms of acute esophagitis during the HA-CRT was a basis for our investigation. An absolute oesophageal volume received a dose of at least 42.8 Gy (within the 95\%-isodose) was identified as a dose-volumetric predictor for the severity of acute oesophageal reaction in treated population. The increase of this volume was strongly correlated with corresponding increase of the CTC-grade and a gradation scale of the likelihood of severity of acute esophagitis was created. Results of our study gain special importance in the scope of the ongoing multicentre randomized phase III trial. Intensification of a neoadjuvant approach due to combi- nation of the chemo- and radiation therapy defines an actual trend in the multimodality treatment of LANSCLC. Prediction of the severity of acute esophagitis based on individual dose-volumetric parameters help to complete overall treatment without interruption. Our gradation scale built a tool for further optimization of radiation therapy treatment planning in LA-NSCLC.

\section{Competing interests}

All authors declare that they have no competing interests.

\section{Authors' contributions}

All authors have made substantial contributions. There is no financial relationship between the authors and commercial companies whose products were used in the study. All authors read and approved the final manuscript.

\section{Author details}

${ }^{1}$ Klinik und Poliklinik für Strahlentherapie und Radioonkologie, Klinikum der Universität München, München, Germany. ${ }^{2}$ Klinik für Thoraxchirurgie; RobertBosch-Hospital, Klinik Schillerhöhe, Stuttgart-Gerlingen, Germany. ${ }^{3}$ Klinik für Strahlentherapie und Radioonkologie, Universitätsklinikum Düsseldorf, Heinrich Heine Universität Düsseldorf, Düsseldorf, Germany.

Received: 22 January 2013 Accepted: 14 May 2013

Published: 17 May 2013

\section{References}

1. Pignon JP, Stewart LA: Randomized trials of radiotherapy alone versus combined chemotherapy and radiotherapy in stages IIIa and IIIb nonsmall cell lung cancer: a meta-analysis. Cancer 1996, 77(11):2413-2414.

2. Saunders M, Dische S, Barrett A, Harvey A, Griffiths G, Palmar M: Continuous, hyperfractionated, accelerated radiotherapy (CHART) versus conventional radiotherapy in non-small cell lung cancer: mature data from the randomised multicentre trial. CHART Steering committee. Radiother Oncol 1999, 52(2):137-148.

3. Turrisi AT 3rd, Kim K, Blum R, Sause WT, Livingston RB, Komaki R, Wagner $H$, Aisner S, Johnson DH: Twice-daily compared with once-daily thoracic radiotherapy in limited small-cell lung cancer treated concurrently with cisplatin and etoposide. N Engl J Med 1999, 340(40):265-271.

4. Curran WJ Jr, Paulus R, Langer CJ Komaki R, Lee JS, Hauser S, Movsas B, Wasserman T, Rosenthal SA, Gore E, Machtay M, Sause W, Cox JD: Sequential vs. concurrent chemoradiation for stage III non-small cell lung cancer: randomized phase III trial RTOG 9410. J Natl Cancer Inst 2011, 103(19):1452-1460.

5. Friedel G, Hruska D, Budach W, Wolf M, Kyriss T, Hurtgen M, Eulenbruch HP, Dierkesmann R, Toomes H: Neoadjuvant chemoradiotherapy of stage III non-small-cell lung cancer. Lung Cancer 2000, 30(3):175-185.

6. Eberhardt W, Wilke H, Stamatis G, Stuschke M, Harstrick A, Menker H, Krause B, Mueller MR, Stahl M, Flasshove M, Budach V, Greschuchna D, Konietzko N, Sack H, Seeber S, et al: Preoperative chemotherapy followed by concurrent chemoradiation therapy based on hyperfractionated accelerated radiotherapy and definitive surgery in locally advanced non-small-cell lung cancer: mature results of a phase II trial. J Clin Oncol 1998, 16(2):622-634.

7. Friedel G, Budach W, Dippon J, Spengler W, Eschmann SM, Pfannenberg C, Al-Kamash F, Walles T, Aebert H, Kyriss T, Veit S, Kimmich M, Bamberg M, Kohlhaeufl M, Steger V, Hehr T: Phase II trial of a trimodality regimen for stage III non-small-cell lung cancer using chemotherapy as induction treatment with concurrent hyperfractionated chemoradiation with carboplatin and paclitaxel followed by subsequent resection: a single-center study. J Clin Oncol 2010, 28(6):942-948.

8. Friedel G, Walles TH: Reply to R. Stupp et al. J Clin Oncol 2011, 29(5):120.

9. Aupérin A, Le Péchoux C, Rolland E, Curran WJ, Furuse K, Fournel P, Belderbos J, Clamon G, Ulutin HC, Paulus R, Yamanaka T, Bozonnat MC, Uitterhoeve A, Wang X, Stewart L, Arriagada R, Burdett S, Pignon JP: Metaanalysis of concomitant versus sequential radiochemotherapy in locally advanced non-small-cell lung cancer. J Clin Oncol 2010, 28(13):2181-2190.

10. Ball D, Bishop J, Smith J, O'Brien P, Davis S, Ryan G, Olver I, Toner G, Walker $\mathrm{Q}$, Joseph D: A randomised phase III study of accelerated or standard 
fraction radiotherapy with or without concurrent carboplatin in inoperable non-small cell lung cancer: final report of an Australian multi-centre trial. Radiother Oncol 1999, 52(2):129-136.

11. Werner-Wasik M, Paulus R, Curran WJ Jr, Byhardt R: Acute esophagitis and late lung toxicity in concurrent chemoradiotherapy trials in patients with locally advanced non-small-cell lung cancer: analysis of the radiation therapy oncology group (RTOG) database. Clin Lung Cancer 2011, 12(4):245-251.

12. Patel $A B$, Edelman MJ, Kwok $Y$, Krasna MJ, Suntharalingam M: Predictors of acute esophagitis in patients with non-small-cell lung carcinoma treated with concurrent chemotherapy and hyperfractionated radiotherapy followed by surgery. Int J Radiat Oncol Biol Phys 2004, 60(4):1106-1112.

13. Hirota S, Tsujino K, Endo M, Kotani Y, Satouchi M, Kado T, Hishikawa Y, Obayashi K, Takada Y, Kono M, Abe M: Dosimetric predictors of radiation esophagitis in patients treated for non-small-cell lung cancer with carboplatin/paclitaxel/radiotherapy. Int J Radiat Oncol Biol Phys 2001, 51(2):291-295.

14. De Ruyck K, Sabbe N, Oberije C, Oberije C, Vandecasteele K, Thas O, De Ruysscher D, Lambin P, Van Meerbeeck J, De Neve W, Thierens H: Development of a multicomponent prediction model for acute esophagitis in lung cancer patients receiving chemoradiotherapy. Int J Radiat Oncol Biol Phys 2011, 81(2):537-544.

doi:10.1186/1748-717X-8-122

Cite this article as: Manapov et al:: Dose-volumetric parameters and prediction of severe acute esophagitis in patients with locallyadvanced non small-cell lung cancer treated with neoadjuvant concurrent hyperfractionated-accelerated chemoradiotherapy. Radiation Oncology 2013 8:122.

\section{Submit your next manuscript to BioMed Central and take full advantage of:}

- Convenient online submission

- Thorough peer review

- No space constraints or color figure charges

- Immediate publication on acceptance

- Inclusion in PubMed, CAS, Scopus and Google Scholar

- Research which is freely available for redistribution 